\title{
To what extent is Denmark vulnerable to mineral supply shortage?
}

\author{
Per Kalvig, Rune J. Clausen, Niels Fold and Karen Hanghøj
}

Mineral resources are building blocks of modern society and essential for progress and prosperity. Mankind has always depended on access to mineral raw materials, which have been a key factor for wealth, culture and development. Modern societies are characterised by a rapidly increasing demand for specialised mineral raw materials, determined by their stage of technological development, the number of consumers, and their standard of living. Generally, the availability of mineral raw materials has not, until recently, been considered an issue by the average consumer or by companies in the downstream end of the value chains, and mineral resources have not been part of the political agenda. In this context China's control over rare-earth elements (REE) has been an eye opener to both industry and politicians worldwide, and has subsequently led to discussions about the possible exhaustion of finite resources and potential threats to the availability of raw materials caused by geopolitical tension and market restrictions.

The increased concern has lead to several attempts to assess the risk of supply shortage which are however still at a rather qualitative stage. Inadequate knowledge about the current and future demand for mineral raw materials prevents political and industrial decision-makers from taking the necessary actions to predict and mitigate the national and industrial vulnerability to supply shortage. Thus, most modern societies, including Denmark, are vulnerable to mineral raw materials scarcity, but unaware of where and how it may appear, and how to prevent and address the problem.

\section{Scarcity issues}

Scarcity issues have been discussed since Thomas Malthus in 1798 initially predicted problems of food shortage due to increasing population and later also in relation to mineral resources. A number of organisations and individuals (e.g. Club of Rome, Gro Harlem Bruntland) have taken the lead in these discussions and emphasised that natural resources are finite and limited and that the global economy is growing disproportionately.

At the summit meeting in Rio de Janeiro in 1992, all nations were encouraraged to adopt the so-called Bruntland principles to ensure sufficient resources for future generations. The term sustainability was introduced to the mining industry. However, no clear effects can be identified neither in the policies nor in the overall mineral consumption, and global and national concern on how to secure raw material supply is increasing. Terms such as critical minerals were introduced, reflecting the risk of scarcity of some raw materials. The US National Research Council quantitatively addressed scarcity issues related to minerals in 2008 (National Research Council 2008), and since then a substantial number of reports have focused on the topic (e.g. Rosenou-Tornow et al. 2009; European Commission 2010; UNDP 2010; Graedel et al. 2012). The decoupling of wealth and mineral resource consumption remains to be seen.

\section{Why are minerals important?}

Mineral-based materials are present everywhere in our daily life - in houses, cars, computers, cooking utensils, paint, tiles, paper, plastic, batteries, wind turbines, roads, pipes etc. For each and all of these 'end products' the choice of raw materials - and thus the minerals that need to be mined depends on the required physical and chemical properties of the products. In some cases more than one material may fulfil the product requirements and the choice will then be based on price and availability.

All societies need mineral resources for their development, but exactly which minerals and metals are in demand and how they are used depend on the stage of development of the particular society. During historic time the trend has been very clear; innovation and new technologies require an increasing number of specialised raw materials. Consequently, we need to explore for new types of minerals to meet new demands.

\section{The demand for minerals is fueled by a number of drivers}

Demographics - The United Nations has estimated that the world population will increase from currently 7 billion to 9 billion by 2050 and that about 6.5 billion people will 
live in cities in 2050. This trend creates a need to develop new infrastructure to support the fast-growing urbanisation, which in turn creates an increased demand for minerals, in particular sand, gravel, iron and copper. Numerous other raw materials are also needed for basic infrastructure.

Wealth - The economic growth in some of the emerging markets - e.g. Brazil, Russia, India, Indonesia, China, the Republic of Korea, South Africa - creates millions of new customers for products like houses, household machines, bicycles, cars, computers, etc. These are all manufactured from raw materials which have to be mined and processed. An example of this is China that has the world's largest population and is globally the largest consumer of copper, aluminium and iron. However, the consumption of copper in China is still only $3 \mathrm{~kg} /$ person/year, much lower than in Europe where the consumption is $16 \mathrm{~kg} /$ person/year (Bogner 2012). However, it is expected that China's copper consumption will increase substantially mainly as a result of growing wealth, rather than just the growing population.

Technology - The introduction of new materials, for example in houses and vehicles, in new electronic communication equipment and in new 'green' energy technology, changes the desired physical and chemical properties of materials, which in turn creates demand for new mineral raw materials. Emerging technologies and new materials have created a rapidly growing demand for certain commodities such as indium and gallium used in light-emiting diode lamps; lithium, copper, neodymium and dysprosium used in electric cars; indium, cadmium and tellurium in photovoltaic thin-film and dysprosium and neodymium in magnets. Concurrently, the need for some traditional materials has been reduced. For example, light, strong materials such as aluminium and magnesium have reduced the amount of steel required to build car frames.

\section{Critical minerals and vulnerability to supply restrictions}

During the past decade mineral resource shortage has made headlines in the media, especially with regard to the REE. In response, a number of institutions have developed lists of mineral criticality on regional and national levels. For example, the European Union has defined 14 raw materials as critical to the EU (European Commission 2010). Typically, the studies have used a two-fold approach: (1) assessment of the supply risk and (2) assessment of the impact of an actual shortage. The term critical minerals is frequently used in this context. Critical minerals are those which are important to

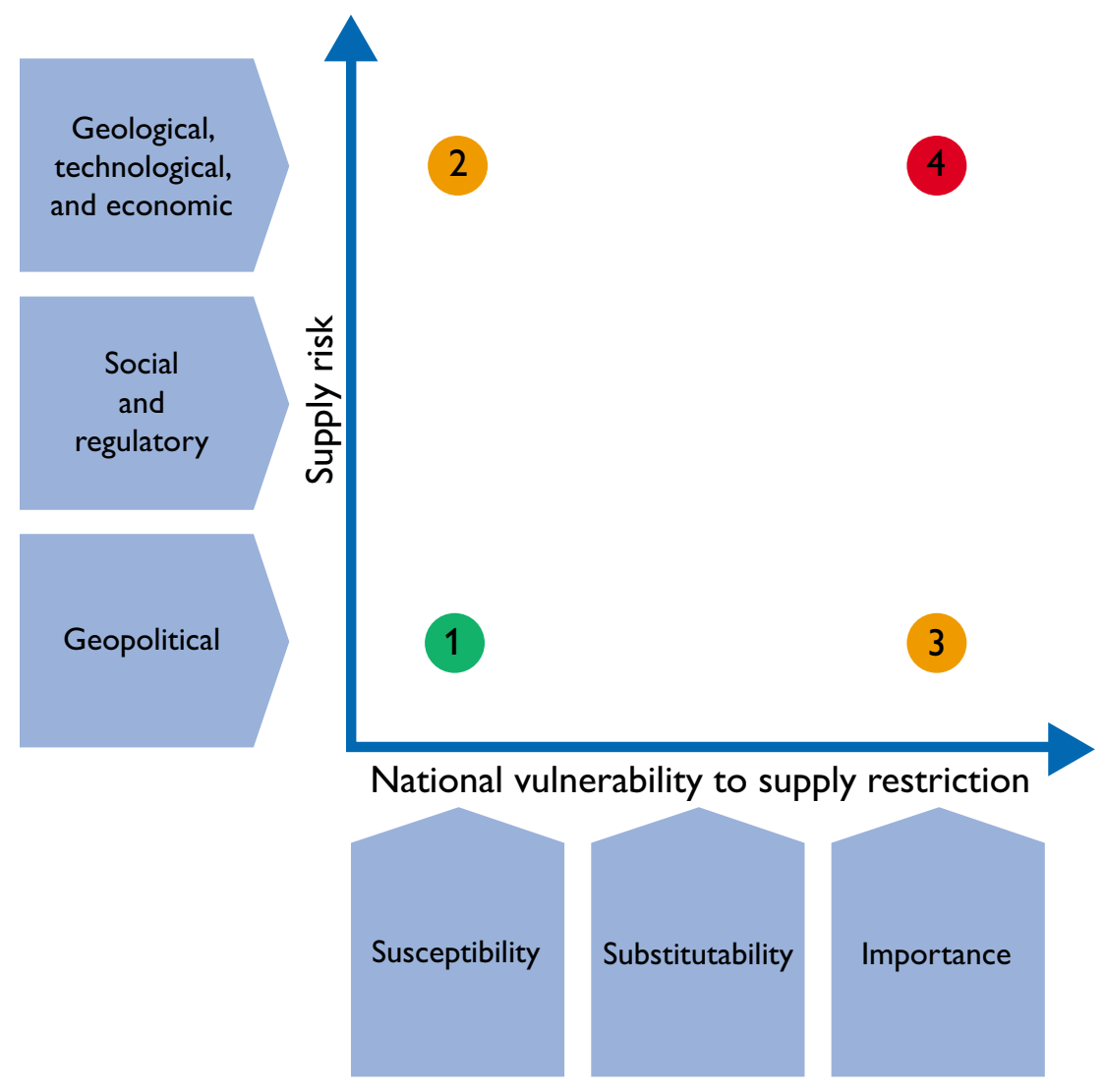

Fig. 1. Diagram of vulnerability to the supply risk and restriction (modified from Graedel $e$ al. 2012). In the diagram element 1 has a low supply risk and even if a supply shortage occurs, this will not have a great impact on society; element 4 has a high supply risk and society is vulnerable to supply restictions; element 2 possesses a high supply risk but low vulnerability to supply restrictions; and for element 3 the opposite situation occurs, the supply risk is low, but in the event of a supply risk the national vulnerability is high. 
society and subject to a specific availability or supply risk, e.g. at the corporate, national, regional or global industry level.

Scarcity is the potential outcome of criticality if a supply risk is not effectively mitigated. Scarcity can be a result of several factors such as political conflicts, embargos, cartels, natural disasters, sudden increases in demand, inadequate investment in new mines and processing facilities or resource depletion. Resource depletion causing significant shortages of mineral commodities has not yet been documented except in the case of cryolite, but it may pose a long-term threat.

Based on long- and medium-term supply risk Graedel et al. (2012) assessed the vulnerability to supply shortage and identified three general components, namely (1) geology, technology and economy; (2) social and regulatory factors and (3) geopolitical factors. Each of these were specified by six indicators, forming the 'supply risk axis'. The 'vulnerability to supply restrictions axis' is composed of another set of factors such as (1) importance, (2) substitutability and (3) susceptibility specified in eight indicators (see Figs 1, 2). Graedel et al. (2012) suggested that vulnerability should also include the environmental impact.

\section{Forecasting and creating possibilities for adequate policies}

The value chains for mineral raw materials include all stages of mineral exploration, mining and the processes transforming the minerals into intermediate goods applicable for manufacturing by industrial end users. However, most of the companies in the chain may be unaware of short- or long-term market constraints or opportunities. This prevents the industry itself from responding to sudden changes in demand. The exploration that targets new raw materials is therefore driven by commodity prices. Globally, 2556 companies spent 20.5 billion US $\$$ on mineral exploration in 2012 , of which $49 \%$ was spent on gold, $32 \%$ on base metals and the remaining $19 \%$ on all other commodities (Wilburn \& Stanley 2013). This illustrates that the exploration sector is decoupled from the end user demand. Furthermore, there is a mismatch between the time scales of action in different parts of the value chain. Industrial demand for new raw materials and markets for raw materials fluctuate on short-time scales, whereas the time needed to adjust the supply is much longer; it typically takes more than ten years to open a new mine, and sometimes even substantially longer. Scrap supplies for recycling, secondary raw materials, are insufficient and usually too expensive to handle in order to bridge the gap between short-term demand and supply.

Individual governments and their institutions need updated assessment data on the national vulnerability to supply restrictions of mineral raw materials in order to develop and implement policies to avoid scarcity of particular critical minerals. For example, the general conditions for Europe may not necessarily be accurate and relevant for the Danish industrial and agricultural sectors. So far, only very limited data on vulnerability to supply restrictions are available for

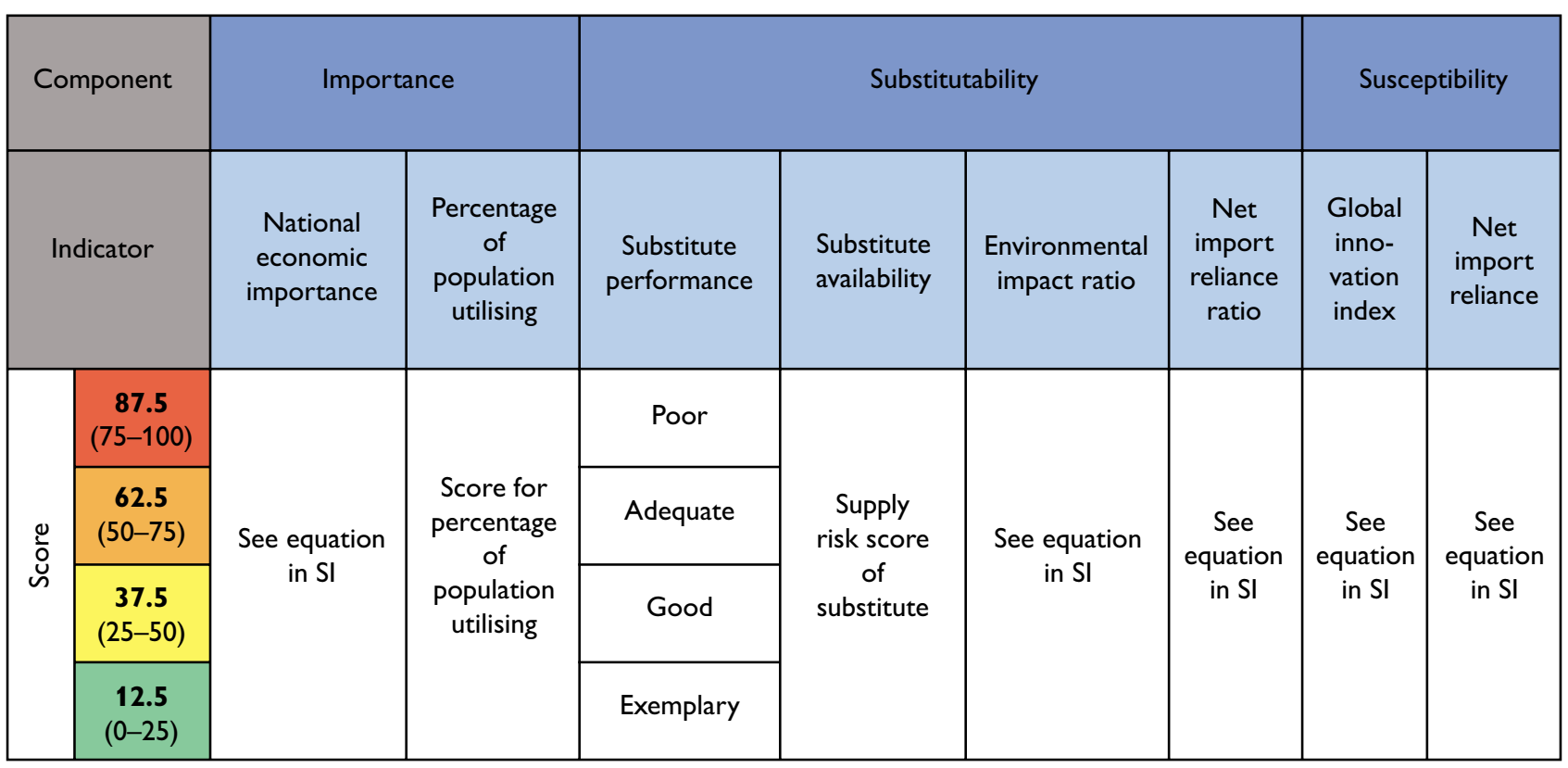

Fig. 2. Components of the valuation methodology for the vulnerability to supply restriction, detailing the X-axis in Fig. 1 (from Graedel et al. 2012). Supporting Information (SI) is detailed in: http://pubs.acs.org/doi/suppl/10.1021/es203534z/suppl_file/es203534z_si_001.pdf 
public and private stakeholders in Denmark. In 2013, the Geological Survey of Denmark and Greenland (GEUS) established the Center for Minerals and Materials (MiMa) to identify and study the most important raw material value chains. The Danish government subsequently decided to strengthen the knowledge about criticality, vulnerability and scarcity of raw materials and have requested MiMa to carry out a three-year research programme to complete a vulnerability analysis for Denmark. MiMa is currently identifying an adequate approach for this programme. Danish industry is characterised by an advanced downstream sector that depends on many imported components in end-product assemblages, while manufacturing of upstream products based on primary raw materials is of lesser importance. However, regardless of where the Danish manufacturing activities belong in the value chains, they are all based on mineral raw materials, some of which may be classified as critical minerals.

It is important to examine and map the extent to which Denmark is subject to supply restrictions and to understand the implications of such vulnerability. Danish consumers may not be aware of a product's requirements with regard to raw materials, and thus remain unaware of a potential supply problem attached to the product. Statistically, Denmark monitors export and import of all goods in compliance with international categories for goods and industries, but there is a need for more knowledge about the amount and types of processed raw materials in these goods and components used by Danish industry. MiMa and its partners will investigate these issues further and disseminate results, analyses and forecasts.

\section{Conclusions}

Denmark, like all other countries, depends on mineral raw materials - domestic and imported - to sustain and develop society and is thus vulnerable to mineral raw materials scarcity. However, most consumers and companies in the downstream parts of the value chains as well as decision makers in the administration and industry are relatively unaware of this. It is the aim of the Center for Minerals and Materials, MiMa, to build knowledge and disseminate information for the Danish society about mineral resource supply risks and vulnerability to supply restrictions.

\section{References}

Bogner, S. 2012: The commodity megatrend. Resource Investor (www.resourceinvestor.com/2012/05/09/the-commodity-megatrend).

European Commission 2010: Critical raw materials for the EU. Report of the ad-hoc working group on defining critical raw materials, $84 \mathrm{pp}$. Brussels: European Commission.

Graedel, T.E. et al. 2012: Methodology of metal criticallity determination. Environmental Science \& Technology 46, 1063-1070.

Malthus, T.R. 1798: An Essay on the Principle of Population, 388 pp. London: J. Johnson.

National Research Council 2008: Minerals, critical minerals, and the U.S. economy. Washington, DC: The National Academies Press.

Rosenau-Tornow, D., Buchholz, P., Riemann, A. \& Wagner, M. 2009: Assessing the long-term supply risks for mineral raw materials - a combined evaluation of past and future trends. Resources Policy 34, 161-175.

UNDP 2010: Human development report 2010 - 20th anniversary edition. The real wealth of nations: pathways to human development, 238 pp. Published for the United Nations Development Programme. Basingstoke: Palgrave MacMillan.

Wilburn, D.R. \& Stanley, K.A. 2013: Exploration review. Annual review 2012. Mining engineering, May 2013, 22-42.

\footnotetext{
Authors' addresses

P.K., R.J.C. \& K.H., Geological Survey of Denmark and Greenland, ØsterVoldgade 10, DK-1350 Copenhagen K, Denmark; E-mail:pka@geus.dk

N.F., Department of Geosciences and Natural Resource Management, Øster Voldgade 10, DK-1350 Copenhagen, K, Denmark.
} 